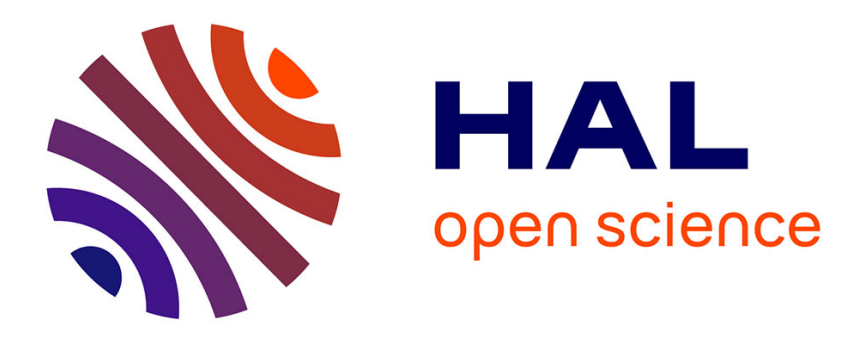

\title{
La traduction du passé prospectif français en polonais
}

Denis Apothéloz, Malgorzata Nowakowska

\section{To cite this version:}

Denis Apothéloz, Malgorzata Nowakowska. La traduction du passé prospectif français en polonais.

Cahiers Chronos, A paraître, Recherches actuelles sur le temps et l'aspect, 32. hal-02503272v1

\section{HAL Id: hal-02503272 \\ https:/ hal.univ-lorraine.fr/hal-02503272v1}

Submitted on 25 Jun 2021 (v1), last revised 17 Sep 2021 (v3)

HAL is a multi-disciplinary open access archive for the deposit and dissemination of scientific research documents, whether they are published or not. The documents may come from teaching and research institutions in France or abroad, or from public or private research centers.
L'archive ouverte pluridisciplinaire HAL, est destinée au dépôt et à la diffusion de documents scientifiques de niveau recherche, publiés ou non, émanant des établissements d'enseignement et de recherche français ou étrangers, des laboratoires publics ou privés. 
À paraître dans : L. Baranzini \& L. de Saussure (éds),

Recherches actuelles sur le temps et l'aspect. Leiden/Boston : Brill, 2021.

(= Cahiers Chronos 32)

\title{
La traduction du passé prospectif français en polonais
}

\author{
Denis Apothéloz, Université de Lorraine \& ATILF \\ Małgorzata Nowakowska, Université Pédagogique de Cracovie
}

\section{Introduction}

Le but de cet article est d'étudier les problèmes de traduction que pose le passé prospectif français quand il s'agit d'en restituer la signification et les diverses nuances en polonais. On appelle ici « passé prospectif» la périphrase verbale < aller à l'imparfait + VINF> lorsque le verbe aller y a un statut d'auxiliaire de conjugaison (comme dans $i l$ allait pleuvoir $)^{1}$. Ces formes sont généralement considérées comme des transpositions dans le passé du futur périphrastique (il va pleuvoir), appelé aussi « présent prospectif ».

La langue polonaise n'ayant pas de temps grammatical pour exprimer la prospectivité (Apothéloz \& Nowakowska 2016), les formes prospectives du français posent de nombreux problèmes quand il s'agit de les traduire dans cette langue. A notre connaissance ces problèmes n'ont, à ce jour, fait l'objet d'aucune étude spécifique. C'est précisément cette lacune que la présente contribution vise à combler. Elle complète une étude antérieure que nous avons consacrée à la traduction polonaise du futur périphrastique français ${ }^{2}$.

Dans un premier temps, nous exposerons les principales difficultés que pose l'analyse de la forme <aller à l'imparfait $+\mathrm{VINF}>$, le passé prospectif n'étant qu'une de ses interprétations grammaticales. Nous examinerons ensuite les propriétés aspectuotemporelles de cette forme quand elle est utilisée pour exprimer le passé prospectif, et distinguerons deux emplois principaux, selon qu'elle met en évidence la phase préparatoire ou la phase processive du procès. Dans un second temps, nous étudierons les possibilités de traduction qu'offre la langue polonaise, en reprenant les différents emplois distingués pour le français. Nous conclurons cette étude par un certain nombre de réflexions sur ce qui distingue les systèmes verbaux du polonais et du français.

\section{La forme < aller à l'imparfait + VINF> et la prospectivité}

Les expressions de la forme <aller à l'imparfait + VINF> peuvent correspondre à plusieurs réalités grammaticales, parfois difficiles à distinguer.

\footnotetext{
${ }^{1}$ Autres appellations : «ultérieur dans le passé » (Damourette \& Pichon 1911-1936), «imparfait prospectif» (Azzopardi \& Bres 2017). Wilmet (1997) le qualifie quant à lui, temporellement de "passé postérieur », et aspectuellement de « sécant perspectif ». Le qualificatif de «prospectif» a été introduit par Benveniste (1959).

${ }^{2}$ Cf. Apothéloz \& Nowakowska (2016). Comme exemple d'autre étude contrastive consacrée à la traduction du passé prospectif français, voir Silletti (2015) pour l'italien.
} 


\subsection{Aller auxiliaire vs aller verbe de déplacement}

Une première distinction à faire est celle entre le passé prospectif, qu'on peut assimiler à un temps verbal ${ }^{3}$ et où aller est un auxiliaire, et les cas où aller est un verbe à statut pleinement lexical. L'exemple (1) est un cas de passé prospectif, et l'exemple (2) un cas où aller a son sens lexical plein de verbe de déplacement.

(1) J'ai pensé à la journée que nous allions avoir, j’y ai pensé avec enthousiasme. (G. Brisac, 1996)

(2) [...] un jour, à Paris, il m'avait croisé dans le métro. C'était une heure de pointe, le wagon était plein. J'allais retrouver Honoré le bureaucrate dans un café de Saint-Michel. (S. Chalandon, 2011)

Cette homonymie fait que la distinction de ces deux interprétations tient parfois à des indices assez subtils. Ainsi, dans (3) l'interprétation de l'expression verbale en gras dans la subordonnée demeure incertaine jusqu'à ce qu'on prenne connaissance de la suite.

(3) Le lendemain, comme il allait faire sa promenade du matin, j'ai eu une idée méritante, je lui ai emboîté le pas. (R. Forlani, 1989)

Avant qu'on prenne connaissance de la dernière proposition (je lui ai emboité le pas), qui suggère que la personne désignée par les pronoms de $3^{\mathrm{e}}$ personne vient juste de partir faire sa promenade, l'expression comme il allait faire sa promenade pourrait signifier aussi bien 'comme il était sur le point de partir faire sa promenade' (passé prospectif) que 'comme il partait faire sa promenade' (avec aller verbe de déplacement). C'est finalement cette dernière interprétation qui doit le plus probablement être retenue, dans la mesure où il ne paraît guère possible d'emboîter le pas à quelqu'un qui n'a pas encore commencé à se déplacer.

Un autre problème d'identification peut tenir au fait que la notion même de déplacement ne concerne pas nécessairement l'espace au sens physique du terme. Il en va ainsi dans l'exemple suivant, où aller signifie bel et bien un déplacement, mais mental et métaphorique :

(4) Moi, écervelée, (car je l'étais à coup sûr), je traitais tout cela de vanité en moi-même, et j’allais chercher l'intimité et la simplicité avec la poésie. (G. Sand, 1855)

Un critère fréquemment évoqué à propos de cette distinction est le fait que, quand il s'agit du passé prospectif, l'auxiliaire aller ne peut être conjugué qu'au présent ou à l'imparfait (il est défectif); tandis que quand aller est un verbe pleinement lexical, il peut être conjugué à tous les temps grammaticaux. Ainsi, on pourrait avoir à la place de (2)

(5) j'allai chercher Lucas chez la nourrice

je suis allé chercher Lucas chez la nourrice

j'étais allé chercher Lucas chez la nourrice

j'irai chercher Lucas chez la nourrice

\footnotetext{
${ }^{3}$ Un problème souvent discuté à propos des formes prospectives est celui de savoir si ce sont des temps verbaux au sens plein du terme ou des périphrases verbales non complètement grammaticalisée. Sur cette question, qu'il n'est pas possible de développer ici, les avis sont divergents. Ainsi, Imbs (1960), Weinrich (1973) ou encore Bres \& Labeau (2012a) considèrent qu'il s'agit de périphrases non complètement grammaticalisées, au contraire de Vet (1993), qui considère que ce sont des temps verbaux au sens plein du terme. Quant à Vetters \& Lière (2009), qui examinent la question en fonction d'un certain nombre de critères de grammaticalisation, ils concluent qu'il s'agit des périphrases aspectuo-temporelles les plus grammaticalisées du français, sans toutefois préciser s'ils les considèrent comme des temps verbaux.
} 
étant allé chercher Lucas chez la nourrice

il faut aller chercher Lucas chez la nourrice

etc.

... ce qui est impossible avec il allait pleuvoir.

\subsection{L'" allure extraordinaire »}

Cependant, une deuxième distinction encore est nécessaire, si l'on veut s'assurer un corpus de données homogène et éviter d'autres confusions. Dans certains emplois, en effet, le verbe aller ne produit pas de sens prospectif, mais plutôt l'idée de dépassement d'une limite dans l'ordre du convenable, du probable ou du vraisemblable (Bres \& Labeau 2012b, 2013). On doit les premières descriptions de cet emploi à Damourette \& Pichon (1911-1936, t.5, §1652-1655), qui lui ont donné l'appellation d'«allure extraordinaire ». Dans les deux exemples ci-dessous, la périphrase est clairement de ce type :

(6) [...] je n'allais tout de même pas leur mâcher le travail. (D. Pennac, 1999)

(7) Si les électeurs allaient en ce moment le nommer empereur! (V. Hugo, 1830)

À la différence du passé prospectif, la périphrase présente alors la particularité d'être compatible avec d'autres temps verbaux que le présent et l'imparfait, comme le montrent les exemples ci-dessous :

(8) La Mannschaft n'a pas réalisé une bonne Coupe du monde, personne n'ira dire le contraire. (sofoot.com, 2018)

(9) Hautemare, fort en colère [...], alla s'imaginer que ce silence était un refus. (Stendhal, 1842)

(10) [...] on est allé jusqu'à proposer de qualifier les emprunts morphologiques de faits de linguistique sociale. (A. Meillet, 1905)

Cet emploi est associé à certaines expressions plus ou moins figées, comme aller chercher dans Ça ira chercher dans les trois mille euros.

On le voit, la séquence <aller à l'imparfait + VINF> peut traduire diverses structures grammaticales, dont n'ont été exposées ci-dessus que les plus fréquentes. La forme grammaticale qui nous retiendra désormais est seulement le passé prospectif et on exclura par conséquent l'«allure extraordinaire » et, bien évidemment, aller verbe de déplacement.

\section{Le passé prospectif : propriétés aspectuo-temporelles et principaux contextes d'emploi}

\subsection{Propriétés aspectuo-temporelles}

Comme le présent prospectif (futur périphrastique), le passé prospectif se caractérise par une référence temporelle sous-déterminée, susceptible de mettre en évidence selon le contexte deux phases distinctes du procès signifié par le lexème verbal : la phase pré-processive (dite aussi "préparatoire ») et la phase processive. La distinction de ces deux interprétations a été repérée depuis longtemps (voir par ex. Gougenheim 1929). Pourtant, selon les formulations et les contextes, elle n'est pas toujours facile à établir. Elle pose le même genre de problèmes, mutatis mutandis, que la distinction des interprétations résultative et processive dans le cas des parfaits. Il est 
cependant assez clair que dans (11), la forme verbale désigne la phase préparatoire, tandis que dans (12), elle désigne la phase processive :

(11) Cinq jours après l'arrestation des gentilshommes, au moment où elle allait se coucher, [...] elle fut appelée dans la cour par sa mère qui arrivait à pied de la ferme. (H. de Balzac, 1846)

(12) Il y avait déjà eu la manif du 1er Mai, dans l'Ouest les syndicats étaient en train de conclure des accords. Bientôt la grève générale allait paralyser Paris et l'ensemble du pays. Des poubelles s'entassaient partout. Plus d'essence. Plus de transports. (J. Kristeva, 1990)

Dans (11), allait se coucher pourrait être remplacé par des formulations comme : était sur le point de se coucher, s'apprêtait à se coucher. Et dans (12), allait paralyser pourrait être remplacé, à sens à peu près équivalent, par des formes comme paralyserait ou devait paralyser (cf. Benveniste 1959), voire paralysera.

Comme avec les parfaits, la distinction des deux interprétations peut tenir à des indices assez subtils. Ainsi, le passé prospectif de (13) est processif en raison de la localisation temporelle indiquée par dans quelques jours, qui localise non la phase préparatoire mais (le début de) la phase processive - d'où la possibilité de remplacer ce passé prospectif par un conditionnel. Mais sans cette indication, la même forme verbale désignerait la phase préparatoire.

(13) Nil allait avoir quarante-trois ans dans quelques jours; Rodin, cinquante-huit en octobre. (G. Matzneff, 1981)

Certains linguistes limitent l'usage du qualificatif de «prospectif» au cas où la forme verbale désigne la phase préparatoire, comme dans (11). La position que nous adoptons est plutôt de considérer que les formes verbales concernées conservent toujours une certaine prospectivité, quand bien même elles désignent la phase processive. Un argument en faveur de cette position est le suivant. Quand, dans une narration comme (12), la forme <aller à l'imparfait + VINF> localise comme ultérieure la phase processive du procès signifié par le verbe à l'infinitif, elle le fait depuis un repère temporel interne au cours narratif, correspondant au moment où est parvenu ce cours. Mais son mode de localisation dans l'ultérieur est différent de celui que produirait, par exemple, un conditionnel ou un futur. Cela tient au fait que, comme l'indiquent Bres et Labeau (2013), le passé prospectif conserve quelque chose de la dynamique du verbe aller : sa prospectivité consiste en un mouvement temporel vers la borne initiale du procès, mouvement dont le point de départ est précisément le repère mentionné plus haut (voir aussi Sarrazin \& Azzopardi 2012). Il s'agit là d'un point important et nous y reviendrons dans la seconde partie de cet article, lorsqu'il s'agira de comparer différentes traductions en polonais. Quoi qu'il en soit, ces considérations nous paraissent suffisantes pour justifier le qualificatif de "prospectif » quelle que soit la phase que la forme verbale met en évidence ${ }^{4}$.

Concernant des exemples comme (11), un autre point doit encore être précisé. Quand il désigne la phase préparatoire, le passé prospectif désigne un intervalle temporel mais ne donne de cet intervalle qu'une représentation partielle. Dans (11), la forme verbale ne désigne pas l'intégralité de la phase préparatoire; elle indique seulement qu'à un certain moment, cette phase est en cours. En un mot, la forme

\footnotetext{
${ }^{4}$ Pour une analyse des différences sémantiques entre le conditionnel et le passé prospectif, voir également Sarrazin \& Azzopardi (2012). Ici encore, il en va des formes prospectives comme des parfaits. De même que les formes prospectives conservent une partie de leur prospectivité quand elles sont interprétées processivement, de même les formes résultatives conservent une partie de leur résultativité quand elles sont utilisées processivement. L'aoriste de discours de Benveniste (1959) n'équivaut pas à un passé simple.
} 
verbale est «sécante ». La possibilité de substituer au passé prospectif un imparfait, sans modification notoire de la référence temporelle (cf. s'apprêtait à se coucher, pour (11)), est un indice tout à fait clair de cette propriété aspectuelle du passé prospectif quand il désigne la phase préparatoire.

\subsection{Principaux contextes d'emploi}

Nous sommes maintenant en mesure d'aborder la question des contextes d'emploi du passé prospectif. Dans la perspective traductologique qui est la nôtre, nous placerons au plus haut niveau la distinction ci-dessus, qui est proprement aspectuelle, et considérerons donc qu'il y a deux grandes familles d'emplois: celle où le passé prospectif désigne la phase préparatoire, et celle où il désigne la phase processive.

\subsubsection{Désignation de la phase préparatoire}

On rencontre ce type de désignation principalement dans trois contextes :

(i) Le premier est celui où la désignation de la phase préparatoire s'accompagne d'une indication selon laquelle la phase processive a été retardée, sinon empêchée (contrefactualité). Cette configuration sémantique correspond à ce qu'on appelle traditionnellement «imminence contrecarrée». Nous verrons qu'elle a certaines conséquences pour la traduction du passé prospectif en polonais.

(14) Il allait refermer la fenêtre lorsqu'il entendit un bruit sourd. (P. Rambaud, 1997)

(15) J'allais faire demi-tour, mais il posa [...] sa main sur mon épaule, et nous voilà en train de marcher côte à côte $[\ldots]$. (H. Bianciotti, 1995)

(16) Il allait demander des explications quand son supérieur ajouta : [...]. (J. Lanzmann, 1994)

(ii) Le deuxième contexte est celui où la désignation de la phase préparatoire n'est accompagnée d'aucune indication concernant la réalisation ou non-réalisation de la phase processive. Exemples :

(17) De deux ans plus âgée [que moi], elle allait avoir quinze ans et n'avait donc encore aucun atout de jeune femme, tout en ayant perdu les avantages de l'enfance [...]. (A. Makine, 1995)

(18) Il y a vingt minutes, je suis descendu boire un double express serré au café du coin qui allait fermer, et acheter un sandwich et deux bières. (P. Sollers, 1993)

(19) Nous nous réveillâmes quand la lumière allait naître ; l'air sentait l'iode ; la mer était là, étale, assoupie ; (H. Bianciotti, 1995)

Notons que dans (14), (15), (16) et (19), l'énoncé rapporte deux procès et localise l'un à l'intérieur de la phase préparatoire de l'autre. Par exemple, dans (14), la perception d'un bruit sourd est localisée temporellement à l'intérieur de la phase préparatoire du procès consistant à refermer la fenêtre ; dans (15), quelqu'un pose sa main sur l'épaule de l'énonciateur au moment où ce dernier s'apprête à faire demi-tour ; etc. Nous verrons que ces configurations temporelles inclusives ont une certaine importance pour la traduction polonaise. La configuration d'imminence contrecarrée est donc un cas particulier de la configuration temporelle inclusive.

Le contexte (i) est fréquemment associé à une construction que la tradition a pris l'habitude d'appeler, d'ailleurs assez improprement, "subordination inverse». Les exemples (14) et (16) illustrent cette construction. Quand et lorsque ne fonctionnent pas 
ici comme des conjonctions mais comme des connecteurs temporels, qu'on peut paraphraser par 'et à ce moment', 'mais à ce moment'.

Dans les exemples ci-dessus, la phase préparatoire est désignée dans le contexte d'une narration. Il en va différemment du troisième contexte.

(iii) Le troisième contexte est celui où l'énoncé comportant le passé prospectif est ancré dans le présent de l'énonciation. On est alors en régime d'« énonciation de discours » et non d' « énonciation historique » :

(20) - Passons maintenant à autre chose, veux-tu ?

- J'allais justement te le proposer. (S. Beckett, 1952)

(21) - Ah ! c'est toi, beugla Boubker [...], en s'introduisant bruyamment dans la cabine [d'un ascenseur]. J'allais justement monter chez toi. (M. Issa, 2016)

(22) les difficultés de langage oral elles se euh _ elles s'organisent je dirais sur une espèce de continuum qui vont des _ d'un retard de langage reta/ relativement simple _ avec un décalage dans les structures d'acquisition _ jusqu'à des difficultés nettement plus complexes et plus _ j'allais dire déviantes mais c'est un petit peu ça quand même _ euh qu'on nomme certaines fois dysphasies (Corpus OFROM, Université de Neuchâtel. Le signe _ indique une pause)

Dans (22), le passé prospectif est associé à une manœuvre qu'on pourrait décrire comme une «prétérition ex post». Cette manœuvre permet de signaler divers problèmes concernant le choix d'un mot ou d'une expression. Elle comporte donc une dimension métalinguistique, mais produit également des effets modaux (Bres et Labeau 2013).

\subsubsection{Désignation de la phase processive}

Nos données indiquent que quand le passé prospectif désigne la phase processive, deux classes d'emplois peuvent être distinguées, selon que la forme verbale est investie ou non d'une signification médiative. Les deux exemples suivants, qui présentent la même expression verbale (allait bientôt partir), permettent de voir de quoi il s'agit.

(23) Après m'être gavée des leçons de Bourg-la-Reine, je pénétrai dans la Sorbonne comme dans un sanctuaire, pleine de respect pour le savoir de mes maîtres. Emile Mâle, qui allait bientôt partir pour diriger l'Ecole française de Rome, nous apprenait à déchiffrer les grands livres de pierre enseignant aux hommes la leçon de Dieu. (J. Bouissounouse, 1977)

(24) Il [le bouvier] ne remonta pas à Journas, il obliqua vers les mélèzes, il suivit la lisière [...]. Il profita d'un recoin d'arbre pour regarder en bas : l'autre ne se souciait pas. Il creusait toujours à coups de hache dans la glace. Le bouvier pensait : « Si on était seulement cinq ou six!»

Mais il fallait aller jusqu'à la ferme et faire vite car l'équipe allait bientôt partir à Villevieille à en juger par l'heure du soleil. (J. Giono, 1934)

L'extrait (23) est tiré d'une séquence de type « récit de vie ». Je pénétrai dans la Sorbonne ne désigne pas ici une entrée singulière et concrète dans la Sorbonne, mais signifie 'je commençai des études à la Sorbonne'. Dans ce contexte, l'expression allait bientôt partir produit une anticipation à l'intérieur du cours narratif. Elle suggère que l'instance narratrice connaît un certain nombre de faits relatifs à l'histoire de la Sorbonne, ce qui lui donne la possibilité de jongler avec la chronologie. C'est donc une prolepse, au sens temporel et narratif que Genette (1972) a donné à ce terme.

Différent est l'extrait (24), qui décrit les pensées d'un personnage (un bouvier) qui se déplace dans un milieu naturel tout en observant quelqu'un en contrebas, désigné ici comme « l'autre ». Le second paragraphe (Mais il fallait aller jusqu'à la ferme et faire 
vite car l'équipe allait bientôt partir...), qui vient immédiatement après une pensée du bouvier rapportée sur le mode du discours direct, doit manifestement être interprété comme la suite de ce rapport de pensée, mais ici sur le mode du discours indirect libre. L'expression à en juger par l'heure du soleil, qui indique la cause de cette pensée, confirme cette interprétation. Le passé prospectif est donc investi, dans cet extrait, d'un sens médiatif - terme que nous empruntons à Guentchéva (1994) : il décrit le point de vue d'un médiateur (le bouvier) auquel est attribué un dire ou une pensée. Il comporte également une dimension proleptique, mais l'anticipation est ici très clairement médiée, attribuée. Il sera intéressant d'examiner quelles sont les conséquences de cette dimension médiative pour la traduction polonaise.

Bres (2012) désigne respectivement par les expressions d' "ultériorité objective » et d' " ultériorité subjective » ces deux types d'anticipation. Quand elle est objective, l'anticipation s'inscrit dans une perspective unilinéaire du cours des événements, de sorte que l'advenue du procès que désigne le passé prospectif est certaine. Mais cette certitude n'est pas une propriété intrinsèque de l'énoncé au passé prospectif. Elle est une conséquence interprétative du contexte particulier où se trouve ce temps verbal, à savoir une narration dans le passé. La validation du procès proleptique est acquise du seul fait qu'il s'agit d'une anticipation dans un passé conçu comme révolu.

Notons également que l'anticipation elle-même, en tant qu'opération de l'instance narratrice, est l'indice d'une intervention de cette instance dans le cours « naturel » des événements. Elle marque ainsi la présence de cette instance. Il en va différemment quand cette opération est subjective (médiative), puisqu'alors, par définition, elle est attribuée à un médiateur; celui-ci en assume la motivation.

Nous avons signalé plus haut que lorsque le passé prospectif désigne la phase processive, il est possible de lui substituer d'autres formes verbales. On peut ajouter maintenant que ces substitutions ne changent rien à la médiativité. Avec des formes habituellement présentées comme plus ou moins équivalentes du passé prospectif, comme < devoir à l'imparfait + VINF> ou un conditionnel, l'extrait (24) n'en serait pas moins médiatif.

Quand il y a médiativité, il est encore possible de distinguer les deux cas suivants : d'une part, ceux où la médiativité intervient dans un contexte de discours (ou de pensée) indirect libre, comme dans (24); d'autre part, ceux où elle intervient dans un contexte de discours (ou de pensée) indirect « lié », donc explicitement rapporté.

En résumé, nous distinguons trois contextes où il y a désignation de la phase processive. Reprenons ces trois contextes, avec quelques exemples additionnels :

- Passé prospectif dans une prolepse simple

(25) La découverte du spin de l'électron, suggérée uniquement par des observations optiques, allait bientôt modifier profondément notre représentation des phénomènes magnétiques. Elle allait d'abord donner la clef d'une énigme qu'avait posée l'étude des effets gyromagnétiques, découverts indépendamment en 1914-1915 par Barnett en Amérique et par Einstein et de Haas en Europe. (Hist. gén. des sciences, 1964)

(26) Rien ne laissait présager qu'une histoire allait se tramer autour de moi et à mon insu, à laquelle, longtemps, j'allais collaborer en toute ignorance [...]. (H. Bianciotti 1995)

(27) Donc, pour augmenter ses revenus, il [l'acteur Cary Grant] se mit à vendre des cravates dans une valise à Broadway. Il se lia d'amitié avec le dessinateur Jack Kelly, qui allait devenir plus tard le styliste Orry-Kelly. (G. Wansell, 1996, trad. franç.)

- Passé prospectif dans un contexte médiatif (type discours indirect libre)

(28) La pharmacienne me dit de repasser le lendemain, elle allait faire le nécessaire pour me trouver le produit original. (H. Guibert, 1990) 
(29) En déplaçant les meubles, ils poussaient des petits rires étouffés, formaient des hypothèses : on allait jouer aux fantômes, ou faire tourner les tables. (E. Carrère, 1995)

(30) Cette offre, si elle attristait mon père, me fit bel et bien danser de joie. J'allais enfin découvrir cette France lointaine, cette terre toujours promise et, surtout, j'allais retrouver Marie-Anne, ma cousine très aimée, ma préférée. (M. de Grèce, 1982)

- Passé prospectif dans un contexte médiatif (type discours indirect lié)

(31) Un soir, il alla chercher ma mère dans sa chambre, l'assit à côté de lui, me demanda de prendre place, et après s'être raclé la gorge annonça à la famille ainsi réunie qu'il allait épouser maman, l'emmener dans sa villa sur la Côte, parmi les pins où crissent les cigales. (P. Roze, 1996)

(32) elle [une boule de neige] devenait de plus en plus grosse en dévalant, énorme, Nicolas devinait_qu'elle allait occuper toute la place, qu'il n'y aurait plus qu'elle et qu'elle l'écraserait. (E. Carrère, 1995)

(33) Le proc [= procureur] comme prévu avait demandé cinq ans et l'interdiction de séjour. Je me demandais s'il allait servir à quelque chose, maître Lubary. (A. Boudard, 1995)

\section{Correspondants du passé prospectif en polonais}

Le polonais n'ayant pas de temps verbal prospectif, il s'agira ici d'examiner quels sont les moyens grammaticaux ou lexicaux dont dispose cette langue, soit pour désigner les phases préparatoire ou processive dans les contextes décrits plus haut, soit pour produire un sens plus ou moins équivalent. Cette dernière précision est importante concernant la phase préparatoire. En effet, si en français il est possible de désigner cette phase, il est également possible de spécifier son mode de déroulement. Par exemple, la phase préparatoire de il allait refermer la fenêtre (ex. 14) peut être lexicalement catégorisée au moyen d'un verbe non auxiliaire, comme dans : il s'apprêtait à fermer la fenêtre, il était sur le point de fermer la fenêtre, il hésitait à fermer la fenêtre. Or, bien évidemment cette possibilité existe aussi en polonais. De même, le sens produit par l'énoncé elle allait avoir quinze ans, interprété comme sélectionnant la phase préparatoire (ex. 17), est finalement très proche de celui que produiraient des formulations comme : sa quinzième année approchait, son quinzième anniversaire était proche, formulations plus «lexicales» que le passé prospectif et qu'on rencontre également en polonais. Dans cette section, on prendra garde par conséquent de bien distinguer, parmi les traductions possibles, celles qui sont proches du passé prospectif français et expriment la phase préparatoire pour ainsi dire « à l'état pur », de celles qui apportent une spécification au contenu de cette phase. De même, s'agissant des interprétations processives, on sera attentif à la dynamique propre à $<$ aller + VINF $>$, dont on a vu qu'elle impliquait un mouvement en direction de la borne initiale du procès, contrairement aux autres expressions de la prospectivité.

\subsection{Désignation de la phase préparatoire}

Selon les cas, le passé prospectif français désignant la phase préparatoire peut être traduit de trois façons en polonais :

(i) par une construction verbale dont le noyau est composé de l'un des deux adverbes już ou właśnie + verbe mieć au passé + VINF,

(ii) par une construction proche de la précédente mais comportant toujours l'adverbe właśnie, 
(iii) par une locution verbo-nominale.

Examinons ces trois traductions.

\subsection{1 <już ou właśnie + verbe mieć au passé + VINF>}

Cette construction intervient typiquement lorsqu'il s'agit de traduire un passé prospectif suivi d'une proposition indiquant que la phase processive a été retardée ou empêchée (imminence contrecarrée). Dans cette configuration, la phase préparatoire est exprimée, en polonais, par une périphrase composée de trois éléments : l'un des deux adverbes już ou właśnie ('justement') + le verbe mieć ('avoir') au passé + VINF. Le procès inclus temporellement dans cette phase et retardant ou empêchant la phase processive est, quant à lui, exprimé par le passé perfectif (souligné, dans les exemples ci-dessous). Les deux procès sont donc exprimés dans une même phrase complexe, qui les met en relation temporelle. Les deux propositions peuvent être liées par gdy/kiedy ('quand') $)^{5}$ ou ale ('mais') $)^{6}$. En voici quelques exemples :

(14) Il allait refermer la fenêtre lorsqu'il entendit un bruit sourd.
(14’) Już/właśnie mial zamykać okno, gdy/kiedy usłyszał ADV avoir.3SG.PASSÉ.IMP fermer.INF fenêtre quand głuchy hałas. entendre.3SG.PASSÉ.PF

(15) J'allais faire demi-tour, mais il posa [...] sa main sur mon épaule, et nous voilà en train de marcher côte à côte $[\ldots]$.
(15') Już/właśnie miałem się obrócić, ale położył swoją dłoń ADV avoir.1SG.PASSÉ.IMP se retourner.INF mais mettre.3SG.PASSÉ.PF sa main na moim ramieniu i już tak szliśmy bok przy boku $[\ldots]$.

Cette traduction est spécifique de la configuration d'imminence contrecarrée. Schématiquement, la formulation (14) est de la forme :

$\mathrm{P}$ (passé prosp.) quand $\mathrm{Q}$

Cependant, la concomitance de $\mathrm{P}$ et de $\mathrm{Q}$ pourrait également être exprimée par les formulations :

quand $\mathrm{P}$ (passé prosp.) Q

quand Q $\mathrm{P}$ (passé prosp.)

$\mathrm{Q}$ quand $\mathrm{P}$ (passé prosp.)

Mais aucune de ces formulations n'est apte à exprimer l'imminence contrecarrée. Conséquence : dans leur traduction en polonais, les adverbes już ou właśnie y sont superflus, voire non naturels :

(14a) Quand il allait refermer la fenêtre il entendit un bruit sourd.

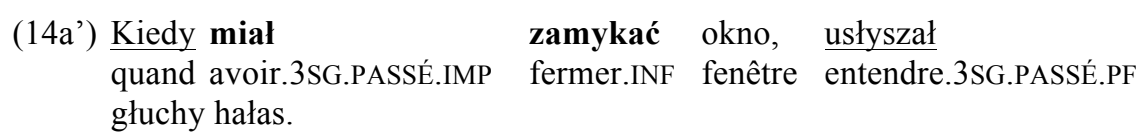

\footnotetext{
${ }^{5}$ Gdy est une variante de kiedy.

${ }^{6}$ Abréviations utilisées dans les gloses métalinguistiques: $\mathrm{V}=$ verbe, PRÉP $=$ préposition, $\mathrm{CONJ}=$ conjonction, $\mathrm{ADV}=$ adverbe $; 1,2,3 \mathrm{SG}$ ou $\mathrm{PL}=1^{\text {ere }}, 2^{\mathrm{e}}, 3^{\mathrm{e}}$ personne du singulier ou du pluriel $; \mathrm{MSC}=$ masculin, $\mathrm{INF}=$ infinitif, $\mathrm{PF}=$ perfectif, $\mathrm{IMP}=$ imperfectif, $\mathrm{PRÉS}=$ présent, $\mathrm{FUT}=$ futur, $\mathrm{COND}=$ conditionnel, IMPERS $=$ impersonnel, DAT $=$ datif.
} 
(14b) Quand il entendit un bruit sourd il allait refermer la fenêtre.

$\begin{array}{llll}\text { (14b’) } & \begin{array}{lll}\text { Kiedy } \\ \text { quand entendre.3SG.PASSÉ.PF } \\ \text { okno. }\end{array} & \begin{array}{l}\text { głuchy hałas } \\ \text { sourd bruit }\end{array} & \begin{array}{l}\text { mial } \\ \text { avoir.3SG.PASSÉ.IMP fermer.INF }\end{array}\end{array}$

(14c) Il entendit un bruit sourd quand il allait refermer la fenêtre.
(14c') Usłyszał głuchy hałas gdy mial zamykać entendre.3SG.PASSÉ.PF sourd bruit quand avoir.3SG.PASSÉ.IMP fermer.INF okno.

La remarque faite plus haut concernant le français est également valable pour le polonais : dans (14'), gdy/kiedy ('quand') n'est pas une vraie conjonction. Comme en français, elle peut être remplacée par un connecteur temporel anaphorique, par exemple $i$ wtedy ('et alors') :
$\begin{array}{llllll}\text { (14”) Już/właśnie } & \text { mial } & \text { zamykać okno i wtedy } & \text { usłyszał } & & \text { alors } \\ & \text { ADV } & \text { avoir.3SG.PASSÉ.IMP } & \text { fermer.INF fenêtre et } & & \text { et }\end{array}$ głuchy hałas.

Et, comme en français également, la conjonction ale ('mais') produirait une interprétation guère différente :

$\begin{array}{lllll}\text { (14’”) Już/właśnie } & \text { mial } & \text { zamykać okno, ale } & \text { usłyszał } \\ \text { ADV } & \begin{array}{l}\text { avoir.3SG.PASSÉ.IMP fermer.INF fenêtre mais } \\ \text { entendre.3SG.PASSÉ.PF }\end{array} & \\ & & & \end{array}$

\subsubsection{Construction : <wlaśnie + mieć au passé + VINF>}

Quand il s'agit d'exprimer la phase préparatoire dans des contextes autres que la configuration temporelle inclusive, l'adverbe wlaśnie est indispensable et ne commute plus avec już. Les exemples (17) et (18) supra se traduisent donc comme suit :

(17) De deux ans plus âgée [que moi], elle allait avoir quinze ans et n'avait donc encore aucun atout de jeune femme, tout en ayant perdu les avantages de l'enfance [...].

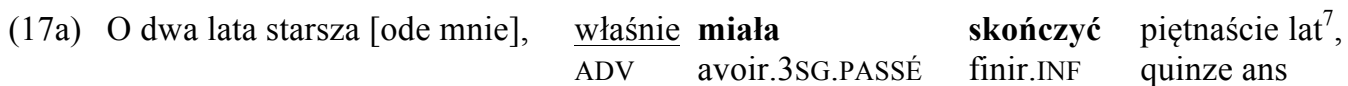
a nie miała jeszcze żadnego atutu młodej kobiety, straciwszy jednocześnie wszystkie dobrodziejstwa dzieciństwa [...].

(18) Il y a vingt minutes, je suis descendu boire un double express serré au café du coin qui allait fermer, et acheter un sandwich et deux bières.

(18a) Dwadzieścia minut temu, poszedłem na podwójne espresso do kawiarni na rogu,

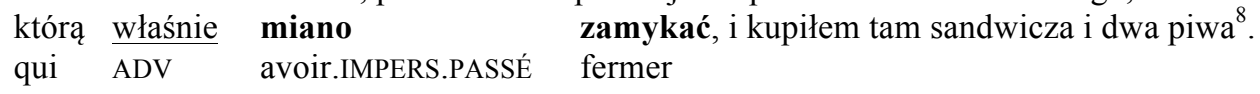

\footnotetext{
${ }^{7}$ Dans la traduction de (17), le verbe n'est pas mieć ('avoir') mais skończyć ('finir'). C'est ainsi qu'on formule l'atteinte d'un âge en polonais.

${ }^{8} \mathrm{La}$ forme miano est une forme impersonnelle passée du verbe mieć formée par la désinence -no.
} 
Si l'adverbe już ne convient pas dans ces formulations, c'est parce que, quand il accompagne <mieć au passé + VINF $>$, il implique l'expression d'un procès inclus dans la phase préparatoire, condition qui n'est pas satisfaite dans les exemples (17a) et (18a).

Cette même construction permet également de traduire le passé prospectif quand l'énoncé est ancré dans le présent de l'énonciation :

(20) - Passons maintenant à autre chose, veux-tu ?

- J'allais justement te le proposer.

(20a) - Przejdźmy teraz do czegoś innego, dobrze? - Właśnie miałem ci to zaproponować. ADV avoir.1SG.PASSÉ te ceci proposer.INF

(21) - Ah ! c'est toi, beugla Boubker [...], en s'introduisant bruyamment dans la cabine [d'un ascenseur]. J'allais justement monter chez toi.

(21a) - Ah ! to ty, zawył Boubker [...], wchodząc hałaśliwie do windy. Właśnie miałem do ciebie wpaść. ADV avoir.1SG.PASSÉ chez toi monter.INF

Dans ces exemples, l'adverbe właśnie est indispensable. Sans lui, la périphrase $<$ mieć + VINF $>$ produit un sens modal.

\subsubsection{Locutions verbo-nominales}

Certaines de ces locutions sont impersonnelles, d'autres non. Commençons par les locutions impersonnelles.

\section{Locutions verbo-nominales impersonnelles}

Ces locutions sont formées d'un verbe conjugué à la $3^{\mathrm{e}}$ personne du passé au genre neutre ${ }^{9}$ et d'un nom abstrait (nom de procès). Leur forme est la suivante :

$\mathrm{V}$ au passé imperfectif, $3^{\mathrm{e}}$ pers. neutre + PRÉP $(n a / k u)+\mathrm{N}$ abstrait

Le nom abstrait correspond au verbe lexical du français. Comme tous les noms abstraits, il peut apparaître sans être complété par les arguments qu'il implique. L'imperfectivité du verbe est conforme à la définition même de la phase préparatoire. Certains verbes de ces locutions n'ont d'ailleurs pas de partenaire perfectif (comme mieć się, lit. 's'avoir').

Pour l'essentiel, quatre verbes interviennent dans ces locutions: mieć się (lit. 's'avoir'), zbierać sie (lit. 'se ramasser'), iść (lit. 'aller à pied'), zanosić się (lit. 's'apporter'). Compte tenu de leur imperfectivité, ces verbes ont un sens qui n'est pas transitionnel.

Voici quelques exemples comportant la préposition $n a$ :

\footnotetext{
${ }^{9}$ En polonais, le temps passé porte la marque du genre du sujet grammatical. Dans ces locutions, il s'agit d'un verbe «phraséologique» car faisant partie de la locution verbale. Ces verbes ne peuvent être conjugués qu'à la troisième personne du singulier au présent et au passé imperfectif. S'agissant de l'impersonnel, ils portent la marque du genre neutre.
} 
(34)

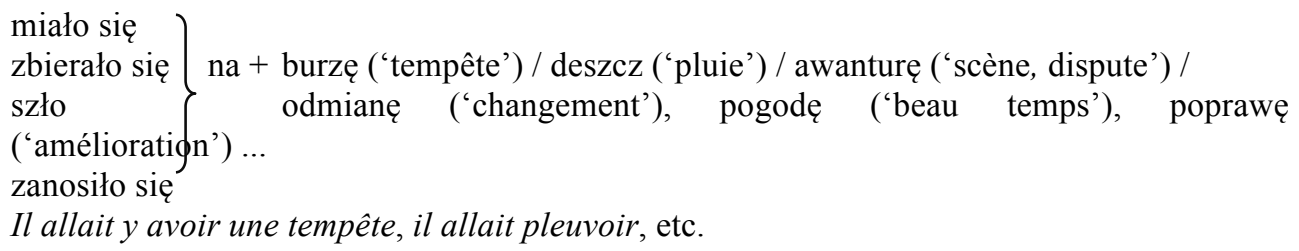

Les noms abstraits de (34) sont mentionnés à titre d'exemples : leur paradigme n'est pas fermé. Ceci dit, il est possible qu'il y ait une certaine conventionnalisation quant à la combinaison de tel verbe avec tel nom abstrait.

La préposition $k u$ se combine quant à elle avec les verbes mieć się et $i s ́ c ́$ :

$$
\left.\begin{array}{l}
\text { miało się } \\
\text { szło }
\end{array}\right\} \begin{aligned}
& \text { lepszemu ('mieux') } \\
& \text { wieczorowi ('soir') } \\
& \text { wiośnie ('printemps') } \\
& \text { zachodowi ('coucher de soleil') } \\
& \text { kolacji ('dîner'), etc. }
\end{aligned}
$$

L'un des verbes mentionnés sous (34), zbierać się (lit. 'se ramasser'), construit une locution qui admet la présence d'un expérienceur. Elle a la forme suivante :

$$
\text { SN DAT / PRONOM DAT + zbierało się }+n a+\mathrm{N} \text { abstrait }
$$

Comme dans (34), le verbe zbierać się est toujours à la forme impersonnelle $\left(3^{\mathrm{e}}\right.$ pers. neutre). Mais ici, la locution comporte un nom ou un pronom au cas datif, représentant l'expérienceur du procès signifié par l'expression $<$ zbierało się $+n a+\mathrm{N}$ abstrait>.

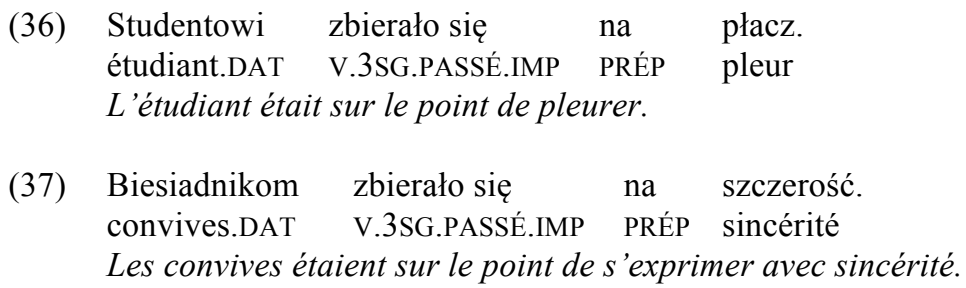

Cette locution indique qu'il se passe quelque chose chez l'expérienceur, le conduisant à accomplir le procès signifié par le nom abstrait ; ou que l'expérienceur est dans un état intentionnel particulier (que spécifie le nom abstrait introduit par $n a)^{10}$.

On notera que la traduction française donnée dans (36a) et (37a) ne correspond pas à la structure polonaise. Elle représente l'argument signifié par un SN ou pronom au datif comme quelqu'un qui va subir le procès signifié par le nom abstrait. De là l'importance du terme expérienceur.

Ces locutions impersonnelles désignent la phase préparatoire et, comme le passé prospectif français, comportent une nuance sémantique de type «mouvement temporel ». Ce sens est dû, d'une part au sens originel des verbes phraséologiques zbierać się (lit. 'se ramasser'), iść (lit. 'aller') et zanosić się (lit. 's'apporter'), d'autre part au sens des prépositions $n a$ et $k u^{11}$. Le verbe mieć się ne comporte en lui-même aucun sens directionnel, mais la présence de la préposition confère à la locution un sens directionnel. Compte tenu de leur structure impersonnelle, toutes ces locutions traduisent donc assez fidèlement le passé prospectif désignant la phase préparatoire.

\footnotetext{
${ }^{10}$ Cf. la définition de zbierać się + na in: Wielki Słownik Języka Polskiego online.

${ }^{11}$ La préposition $n a$ régit ici l'accusatif et a un sens directionnel (dans d'autres emplois elle peut régir le locatif). La préposition $k u$ régit quant à elle le datif et a également un sens directionnel.
} 


\section{Locutions verbo-nominales personnelles}

Examinons maintenant le cas des locutions verbo-nominales personnelles. Leur verbe phraséologique n'a pas de marque de genre neutre et n'est pas limité à la $3^{\mathrm{e}}$ personne du singulier. Il s'accorde en genre et en nombre avec le sujet. Il existe deux locutions de ce type : la première est utilisée lorsque le sujet est [+intentionnel], la seconde lorsqu'il est [-intentionnel].

La première locution comporte à nouveau le verbe zbierać się (qui sert à former l'une des locutions impersonnelles ci-dessus), mais le nom abstrait est ici introduit par la préposition $d o$. Compte tenu de la sélection qui s'exerce sur le type de sujet, cette locution ne pourrait pas traduire un énoncé comme Le train allait partir. Elle pourrait convenir en revanche dans le cas suivant :

$\begin{array}{llll}\text { (38) Piotr } & \text { zbierat się } & \text { do } & \text { wyjścia. } \\ \text { Pierre } & \text { V.3SG.PASSÉ.IMP } & \text { PRÉP. } & \text { sortie } \\ \text { Pierre s'apprêtait à sortir. } & \end{array}$

Toutefois, le verbe zbierać się a un sens plus spécifique que dans la locution précédente. Il équivaut à peu près, en français, à s'apprêter à. Il confère donc un contenu à la phase préparatoire et pour cette raison ne traduit qu'imparfaitement le passé prospectif français.

La seconde locution (à sujet [-intentionnel]) se compose du verbe zapowiadać się (lit. 's'annoncer') et de la préposition $n a$ suivie d'un nom abstrait :

(39) Pogrzeb zabitych robotników
$\begin{aligned} & \text { zapowiadal się } \\ & \text { V.3SG.PASSÉ.IMP }\end{aligned}$
$\begin{aligned} & \text { L'enterrement des ouvriers } \\ & \text { manifestation. }\end{aligned}$

Etant donné que cette locution implique un sujet [-intentionnel], (39) signifie que, compte tenu de ce que l'on savait du déroulement des événements, il était attendu que l'enterrement des ouvriers donne lieu une grande manifestation ${ }^{12}$. Le verbe zapowiadać się ajoute donc ici encore un sens supplémentaire, absent dans le passé prospectif français.

Contrairement aux locutions impersonnelles, les deux locutions personnelles ont ainsi un sens plus spécifique que la simple indication de la phase préparatoire.

\subsubsection{Faits résiduels}

Comme nous l'avons vu en 2.2.1, le passé prospectif peut être utilisé pour signifier une prétérition ex post (cf. j'allais dire, j'allais écrire, etc.) ${ }^{13}$. Mais ce type d'expression se traduit, en polonais, par un verbe conjugué au conditionnel: respectivement, powiedziałbym ('je dirais'), napisałbym ('j'écrirais'). Reprenons un exemple donné plus haut, dont nous ne traduisons que la séquence concernée :

(22) les difficultés de langage oral elles se euh _ elles s'organisent je dirais sur une espèce de continuum qui vont des _ d'un retard de langage reta/ relativement simple _ avec un décalage dans les structures d'acquisition _ jusqu'à des difficultés nettement plus complexes et plus _ j'allais dire déviantes mais c'est un petit peu ça quand même _ euh qu'on nomme certaines fois dysphasies (Le signe _ indique une pause)

\footnotetext{
${ }^{12}$ Cf. la définition de zapowiadać się in: Wielki Słownik Języka Polskiego online.

${ }^{13}$ Ces expressions servent à marquer une sorte de distance vis-à-vis du choix d'un mot ou d'une expression, et ont des points communs avec pour ainsi dire (pol. że tak powiem). Mais avec le passé prospectif, l'idée de distance vient de la désignation même de la phase préparatoire, qui laisse entendre que le procès proprement dit, consistant à utiliser telle ou telle expression, n'est pas advenu.
} 
(22a) powiedziałbym niepoprawne

dire.1SG.COND.PF.MSC déviantes

a to trochę tak jest mimo wszystko jak to się nazywa czasami dysfazją

La traduction polonaise perd ici toute idée de phase préparatoire, de même d'ailleurs que toute idée de prétérition. Elle conserve en revanche - et peut-être même accentue - le caractère modal de la formulation française.

Notons qu'en polonais, le morphème de conditionnel comporte une indication du genre grammatical du sujet. Dans (22a), c'est la forme masculine que nous avons indiquée (dans la glose: MSC); la forme féminine correspondante serait powiedziałabym.

\subsection{Désignation de la phase processive (prolepse)}

Examinons successivement le cas de la prolepse en contextes médiatif et non médiatif.

\subsubsection{Contexte médiatif : futur polonais}

Comme nous l'avons vu en 2.2.2, le passé prospectif proleptique est susceptible de produire des effets médiatifs lorsqu'il apparaît dans un contexte de discours ou de pensée rapportés («ultériorité subjective» de Bres 2012). En français, le repérage énonciatif en discours indirect, en ce qui concerne la personne et le temps verbal, est le plus souvent réglé sur celui de l'instance citante. Mais il en va autrement en polonais. Dans cette langue, le repérage énonciatif, en ce qui concerne le temps verbal, est toujours celui de l'instance citée. Le passé prospectif proleptique français en discours indirect est donc traduit en polonais par le futur ${ }^{14}$. Exemples :

(31) Un soir, il alla chercher ma mère dans sa chambre, l'assit à côté de lui, me demanda de prendre place, et après s'être raclé la gorge annonça à la famille ainsi réunie qu'il allait épouser maman, l'emmener dans sa villa sur la Côte, parmi les pins où crissent les cigales.

(31a) Pewnego wieczoru, poszedł po matkę do sypialni, posadził ją obok siebie, poprosił mnie, żebym zajął miejsce, odchrząknął i ogłosil zebranej rodzinie,

że poślubi mamę, i ją zabierze.

que épouser.3SG.FUT.PF maman et la emmener.3SG.FUT.PF

na Wybrzeże do swojej willi, znajdującej się wśród sosen, gdzie roznosi się dźwięk cykad.

(29) En déplaçant les meubles, ils poussaient des petits rires étouffés, formaient des hypothèses : on allait jouer aux fantômes, ou faire tourner les tables.

(29a) Przesuwając meble, tłumili wybuchy śmiechu i zastanawiali się:

będą się bawić $\quad$ w duchy czy w seans spirytystyczny.

jouer.3PL.FUT.IMP

Toutefois, si le futur polonais dans ce contexte rend correctement la prolepse dans sa dimension d'ultériorité, il ne rend pas compte de la dynamique propre au passé prospectif français, consistant en un mouvement temporel en direction de la borne initiale du procès. Selon le contexte, le futur polonais serait ici comparable à un conditionnel ou à un futur français (cf. respectivement ex. (31) et (29)).

On notera que dans (29), la médiativité du passé prospectif a des implications sur l'interprétation de l'indéfini on. Si ce pronom est interprété comme excluant la $1^{\text {ère }}$

\footnotetext{
${ }^{14}$ Le polonais possède un futur perfectif et un futur imperfectif. Le choix de l'une des deux formes n'est pas pertinent pour le problème traité ici.
} 
personne, le passé prospectif n'est pas médiatif. Une interprétation médiative du passé prospectif implique la possibilité d'interpréter on comme équivalent à un pronom de 1 ère personne du pluriel. En polonais, où ce type de pronom n'existe pas, c'est l'utilisation de la $3^{\mathrm{e}}$ personne du pluriel couplée avec le futur, comme dans (29a), qui marque le discours indirect libre. La $1^{\text {ère }}$ personne du pluriel marquerait ici un discours direct.

\subsubsection{Absence de contexte médiatif : <mieć au passé + VINF $>$ ou futur du V lexical}

Le passé prospectif proleptique sans effet médiatif ("ultériorité objective ») peut avoir deux traductions : la périphrase <mieć au passé + VINF $>$ ou le futur du verbe lexical. Examinons d'abord le premier cas. Voici la traduction de l'exemple (27) donné supra et d'un extrait de biographie :

(12) Il y avait déjà eu la manif du 1er Mai, dans l'Ouest les syndicats étaient en train de conclure des accords. Bientôt la grève générale allait paralyser Paris et l'ensemble du pays. Des poubelles s'entassaient partout. Plus d'essence. Plus de transports.

(12a) Była już manifestacja pierwszego maja, a na zachodzie związki zawodowe właśnie podpisywały porozumienie. sparaliżować Paryż i cały kraj.

bientôt grève générale avoir.3SG.PASSÉ paralyser.INF Śmieci w stosach kłębiły się wszędzie. Brak benzyny i transportu.

(27) Donc, pour augmenter ses revenus, il [1'acteur Cary Grant] se mit à vendre des cravates dans une valise à Broadway. Il se lia d'amitié avec le dessinateur Jack Kelly, qui allait devenir plus tard le styliste Orry-Kelly.

(27a) Więc, żeby powiększyć swoje zarobki, zaczął sprzedawać na Broadwayu krawaty z walizki. Zaprzyjaźnił się z rysownikiem Jackiem Kelly,

który później mial stać się stylistą o nazwisku Orry-Kelly.

qui plus tard avoir.3SG.PASSÉ devenir.INF

On notera que, contrairement à la solution suivante (verbe lexical au futur), le passé du verbe mieć est ici en accord avec le temps du contexte de la narration. Dans cet emploi, la périphrase <mieć au passé + VINF> signifie bien une anticipation dans le passé et, à cet égard, traduit correctement le passé prospectif français.

Cependant, cette périphrase comporte également un sens modal : elle signifie la nécessité aléthique (cf. Topolińska 1968/2008, Lempp 1986: 80-81, Holvoet 2012 : 140-143, Hansen 2001: 137; 2015: 168-169, Nowakowska 2017). Cette modalité a de surcroît ici une valeur particulière, évidentielle : elle signale que l'instance narratrice n'est pas la source de l'information et qu'elle délègue la validation du procès à une autre instance (cf. Topolińska 2000/2008). Quelle est cette instance ?

Berrendonner (1981 : 35-73) a montré que diverses instances peuvent valider un énoncé : le locuteur, l'opinion commune (la doxa), mais aussi l'univers référentiel luimême, autrement dit l'" ordre des choses » (voir aussi Gosselin 2010 : 61-62). Nous pensons que c'est précisément à ce type de validation que nous avons affaire dans les exemples (12a) et (27a). Par son sens évidentiel, la périphrase polonaise déplace la validation du procès de l'instance narratrice à l'univers référentiel. La conséquence est que ce procès est présenté non seulement comme advenu, mais nécessairement advenu $^{15}$. La nécessité aléthique est donc ici la conséquence d'un type tout à fait

\footnotetext{
${ }^{15}$ Cette analyse de la périphrase <mieć au passé + VINF> confirme les intuitions de certains chercheurs qui appellent ce sens « destiny » ou, comme le fait Hansen (2001: 133-137), « fatalistiches Futur in der Vergangenheit ». Cette analyse rejoint également celle que fait Benveniste de la périphrase latine $<$ habēre (à l'imparfait) + VINF> : cette périphrase avait, selon lui, la fonction "d'indiquer la prédestination » (1974 : 132).
} 
particulier d'évidentialité. Cette valeur est totalement absente du passé prospectif français.

Le passé prospectif proleptique en contexte non médiatif peut également être traduit en polonais par le futur du verbe lexical. Reprenons les deux exemples cidessus :

(12) Bientôt la grève générale allait paralyser Paris et l'ensemble du pays.

(12b) Wkrótce strajk generalny sparaliżuje Paryż i cały kraj.

bientôt grève générale paralyser.3SG.FUT

(27) Il se lia d'amitié avec le dessinateur Jack Kelly, qui allait devenir plus tard le styliste OrryKelly.

(27b) Zaprzyjaźnił się z rysownikiem Jackiem Kelly,

który później stanie się stylistą o nazwisku Orry-Kelly.

qui plus tard devenir.3SG.FUT

Contrairement à la traduction précédente, celle qui utilise le futur ne doit son interprétation d'ultériorité dans le passé qu'au contexte dans lequel elle se trouve (narration dans le passé). On pourrait la rapprocher d'un futur narratif français. De ce point de vue, son fonctionnement interprétatif est différent de la traduction précédente ainsi que du passé prospectif français.

\section{$5 \quad$ Bilan et conclusion}

Dans un célèbre article consacré à la traduction, R. Jakobson écrivait que « les langues diffèrent essentiellement par ce qu'elles doivent exprimer, et non par ce qu'elles peuvent exprimer. " (Jakobson 1959/1963 : 84). Les problèmes que pose la traduction en polonais du passé prospectif français sont une bonne illustration de cet axiome. Essayons de faire la synthèse de ces problèmes.

Tout d'abord, il convient de rappeler que la langue polonaise ne comporte aucun temps verbal prospectif. Il n'est donc pas surprenant de trouver, dans cette langue, des locutions verbales ou des constructions plus ou moins figées qui pallient ce manque et dont le sens équivaut à celui que produit le passé prospectif français. De façon générale, nous avons constaté que ces locutions et constructions permettent, selon le contexte, de rendre assez fidèlement les deux emplois majeurs du passé prospectif : celui consistant à désigner la phase préparatoire, et celui consistant à désigner la phase processive (prolepse). Cependant, au-delà de cette distinction, et si l'on regarde les choses plus en détail, on s'aperçoit que le passé prospectif français a développé certains emplois dont la traduction polonaise "fidèle » est pratiquement impossible, pour des raisons qui tiennent précisément à l'axiome de Jakobson rappelé ci-dessus. Ce cas de figure est illustré par quatre situations dégagées dans les analyses ci-dessus.

La première est celle que nous avons rencontrée avec la traduction de la phase préparatoire. Pour le passé prospectif français, cette phase est un intervalle temporel vide de tout contenu, ayant pour seule caractéristique de précéder (plus ou moins immédiatement) le procès proprement dit. Or, nous avons vu qu'en polonais, cette phase doit parfois être traduite au moyen de locutions verbo-nominales qui lui confèrent un certain contenu : l'une de ces locutions, par exemple, a un sens s'approchant du français s'apprêter à. Dans ce cas, le polonais contraint à catégoriser lexicalement la phase préparatoire et produit ainsi un sens plus spécifique que le passé prospectif français.

La deuxième situation est celle, tout à fait particulière, où le français utilise le passé prospectif pour marquer une certaine distance vis-à-vis du choix d'un mot ou 
d'une expression (type : j'allais dire...), produisant ainsi une prétérition ex post. Ce type d'énoncé ne peut être restitué en polonais que par un conditionnel, de sorte que cette langue ne traduit ici que la dimension modale de l'expression française, non sa dimension aspectuo-temporelle. Cet exemple est intéressant car il montre que l'une des vertus de l'exercice de la traduction est d'agir comme un révélateur de la phraséologie et des faits de pur usage : au regard de la langue polonaise, le passé prospectif français utilisé pour signifier la prétérition ex post apparaît comme une sorte de figure.

Une troisième situation tout à fait caractéristique de la différence entre le français et le polonais concerne la gestion du repérage énonciatif. Dans les séquences de discours ou de pensée rapportés, le polonais exprime la simultanéité par le présent, l'antériorité par le passé et la postériorité (ultériorité) par le futur, adoptant le point de vue temporel du discours cité et non du discours citant. Lorsqu'il s'agit de traduire le passé prospectif proleptique dans un contexte médiatif, le polonais utilise par conséquent le futur. Ce temps verbal est d'ailleurs également possible quand il s'agit d'exprimer une prolepse en contexte non médiatif. Le futur doit alors sa valeur proleptique au contexte passé dans lequel il se trouve. Mais, dans les deux cas, il ne rend pas compte de la dynamique temporelle propre au passé prospectif. On peut déduire de ce fonctionnement que le futur français est plus marqué déictiquement que le futur polonais.

La quatrième situation où la traduction du passé prospectif fait problème est, en contexte non médiatif, la construction polonaise <mieć au passé + VINF $>$. Cette construction évite certes les problèmes que pose le futur (notamment parce qu'elle ne quitte pas le passé), mais comporte une valeur évidentielle et modale tout à fait particulière : elle indique que l'énonciateur ne prend pas en charge la validation du procès et délègue cette opération à une instance externe, qui n'est autre que la réalité elle-même, l'univers référentiel. Il en résulte une modalité de nécessité aléthique (ce que Benveniste appelait «prédestination »). Cette signification est absente du passé prospectif français, la validation du procès de la prolepse y étant pleinement assumée par le narrateur, et de toute manière acquise, du seul fait qu'il s'agit d'une anticipation dans un passé par convention révolu. La dimension évidentielle est donc absente en français. Ici encore, on s'aperçoit que la langue polonaise oblige à produire, en plus de la prolepse, des significations qui n'existent pas en français.

Quelques faits moins saillants sont également apparus au cours de cette étude. Celui que nous mentionnerons dans cette conclusion concerne les expressions polonaises verbo-nominales comportant un nom (ou son substitut pronominal) au cas datif, représentant un expérienceur. Ces expressions nous indiquent indirectement que, en français, des formulations comme il allait éclater en sanglot et il allait sortir, formellement identiques, attribuent un rôle sémantique différent au sujet syntaxique : celui-ci est dans la situation de subir un procès dans le premier exemple, ce qui suppose une cause externe ; tandis qu'il est agent et lui-même la cause du procès dans le second exemple. Or, cette différence est grammaticalement marquée en polonais, de sorte que, ici encore, on trouve une illustration de l'axiome de Jakobson.

Terminons par une dernière remarque, qui concerne l'expression <mieć + VINF>. On a vu que cette expression intervient souvent dans la traduction du passé prospectif. Mais son sens premier est modal: aléthique, déontique ou volitif (boulique). Pour qu'elle puisse désigner la phase préparatoire, elle doit être associée à un type particulier d'adverbe, dont le sens est proche de juste ou de précisément en français, adverbe qui a pour effet de suspendre la sens modal. Sans cet adverbe, elle peut également signifier, dans le cours d'une narration, un procès anticipé (prolepse), mais donne à cette anticipation un sens évidentiel et modal particulier. 


\section{Références}

Apothéloz, Denis \& Nowakowska, Małgorzata. 2016. Comment traduire le futur périphrastique français en polonais ? In : Inkova, Olga \& Trovesi, Andrea (éds), Langues en contraste / Славянские языки in comparatione / Lingue a confronto, 5583. Bergamo University Press : Sestante Edizioni.

Azzopardi, Sophie \& Bres, Jacques. 2017. Le système temporel et aspectuel des temps verbaux de l'indicatif (en français). Verbum 39/1. 71-112.

Benveniste, Emile. 1959/1966. Les relations de temps dans le verbe français. Bulletin de la Société de Linguistique 54/1. Réédité dans : Problèmes de linguistique générale 1, 237-250. Paris : Gallimard, 1966.

Benveniste, Emile. 1968/1974. Mutations of linguistic categories. In : Lehmann, Winfred P. \& Malkiel, Yakov (eds), Directions for historical linguistics: A symposium, 85-94. Austin: University of Texas Press. Version française: Les transformations des catégories linguistiques. In : Problèmes de linguistique générale 2, 126-136. Paris : Gallimard, 1974.

Berrendonner, Alain. 1981. Éléments de pragmatique linguistique. Paris : Les Éditions de Minuit.

Bres, Jacques. 2012. Conditionnel et ultériorité dans le PASSÉ: de la subjectivité à l'objectivité. $3^{\text {ème }}$ Congrès Mondial de Linguistique française (CMLF 2012), Lyon, 4-7 juillet 2012. https://doi.org/10.1051/shsconf/20120100037

Bres, Jacques \& Labeau, Emmanuelle. 2012a. De la grammaticalisation des formes itive (aller) er ventive (venir) : valeur en langue, emplois et discours. In : de Saussure, Louis \& Rihs, Alain, Études de sémantique et pragmatique françaises, 143-165. Berne : P. Lang.

Bres, Jacques \& Labeau, Emmanuelle. 2012b. Allez donc sortir des sentiers battus ! La production de l'effet de sens extraordinaire par aller et venir. Journal of French Language Studies 23 (2). 151-177.

Bres, Jacques \& Labeau, Emmanuelle. 2013. Aller et venir : des verbes de déplacement aux auxiliaires aspectuels-temporels-modaux. Langue française 179. 13-28.

Damourette, Jacques \& Pichon, Edouard. 1911-1936. Des mots à la pensée. Essai de grammaire de la langue française, Tome 5. Paris : J.-L.-L. d'Artrey.

Genette, Gérard. 1972. Figures III. Paris : Ed. du Seuil.

Gosselin, Laurent. 2010. Les modalités en français. La validation des représentations. Amsterdam : Rodopi.

Gougenheim, Georges. 1929. Étude sur les périphrases verbales de la langue française. Paris: Les Belles Lettres.

Guentchéva, Zlatka. 1994. Manifestations de la catégorie du médiatif dans les temps du français. Langue française 102. 8-23.

Hansen, Bjørn. 2001. Das slavische Modalauxiliar: Semantik und Grammatikalisierung im Russischen, Polnischen, Serbischen/Kroatischen und Altkirchenslavischen. München: Sagner.

Hansen, Bjørn. 2015. Opis polskich czasowników i predykatów modalnych w słowniku walencyjnym. In: Roszko, Danuta \& Satoła-Staśkowiak, Joanna (eds), Semantyka a konfrontacja językowa, vol. 5, 161-171. Warszawa: SOW (Slawistyczny Ośrodek Wydawniczy).

Holvoet, Axel. 2012. Polish mieć and the semantic map of interpretive deontics. Zeitschrift für Slawistik 57/2. 129-146.

Imbs, Paul. 1960. L'emploi des temps verbaux en français moderne. Paris : Klincksieck. Jakobson, Roman. 1959. On linguistic aspects of translation. In : Brower, Reuben A. (ed.), On translation, 232-239. Harvard University Press, Trad. franç. : Aspects 
linguistiques de la traduction. In : Essais de linguistique générale 1, 78-86. Paris : Ed. de Minuit, 1963.

Lempp, A. (1986). Mieć. 'To have' in modern Polish. München: Verlag Otto Sagner.

Nowakowska, Małgorzata. 2017. Interpretacja prospektywna peryfrazy <mieć + bezokolicznik>. Biuletyn Polskiego Towarzystwa Językoznawczego VXXIII (Bulletin de Société Polonaise de Linguistique VXXIII). 221-246.

Sarrazin, Sophie \& Azzopardi Sophie. 2012. L'alternance du conditionnel et de la périphrase itive à l'imparfait dans des corpus oraux espagnols et français. Studia Universitatis Babes-Bolyai - Philologia 57/3. 57-68.

Silletti [orthographié par erreur «Silleti »], Alida Maria. 2015. 'Allait + infinitif' et sa traduction en italien : imminence et/ou futur dans le passé ? Cahiers de praxématique 65. URL : http://journals.openedition.org/praxematique/4192

Topolińska, Zuzanna. 1968/2008. Miejsce konstrukcji z czasownikiem mieć w polskim systemie werbalnym. Studia Orientalis 17/3. 427-431 (reprint In: Topolińska, Zuzanna. Z Polski do Macedonii, vol 1. 24-29. Kraków: LEXIS).

Topolińska, Zuzanna. 2000/2008. „Dystans” - informacja zgramatykalizowana w polskim systemie werbalnym? In: Mindak, Jolanta \& Wrocławski, Krzysztof (eds): Folia Philologica Macedono-Polonica, tom 5, 86-93. Warszawa: UW, (reprint In: Zuzanna Topolińska Z Polski do Macedonii, vol 1. 286-292. Kraków : LEXIS).

Vet, Co. 1993. Conditions d'emploi et interprétation des temps futurs du français. Verbum 4. 71-84.

Vetters, Carl \& Lière, Audrey. 2009. Quand une périphrase devient temps verbal : le cas d'aller + infinitif. Faits de langues 33. 27-36.

Weinrich, Harald. 1973. Le temps. Paris : Ed. du Seuil. (Tempus. Stuttgart: W. Kohlhammer, 1964)

Wilmet, Marc. 1997. Grammaire critique du français. Bruxelles : Duculot. 